\title{
Endovascular Aneurysm Repair and Sealing (EVARS): A Useful Adjunct in Treating Challenging Morphology: Response to Comments
}

\author{
Gareth J. Harrison $^{1} \cdot$ George A. Antoniou ${ }^{1} \cdot$ Francesco Torella $^{1}$. \\ Richard G. McWilliams ${ }^{1}$ - Robert K. Fisher ${ }^{1}$
}

Received: 20 April 2016/Accepted: 26 April 2016/Published online: 31 May 2016

(C) Springer Science+Business Media New York and the Cardiovascular and Interventional Radiological Society of Europe (CIRSE) 2016

To the Editor,

We would like to thank the commentators for their interest in our paper [1]. EVARS as described in our report uses the standard 2 stent EVAS system as the basis for repair of a complex aneurysm with additional support of standard EVAR iliac limbs. This is a practical combination based on the conventional use of existing technologies; EVARS described by us, currently, is only an option to treat difficult anatomy rather than a further endovascular revolution.

$\mathrm{Bi}$ et al. propose the development of a system using a single endobag around a bifurcated stent termed EVARS $[2,3]$. There are potential attractions to such a proposal in the avoidance of type 2 endoleaks and possibly in conformability to difficult-shaped aortic necks. However, there would be difficulties deploying such a bifurcated device, which would have to be in one piece for filling of the single endobag, in contrast to all commercially available EVAR devices. In addition, in our experience with EVAS, the second endobag has avoided difficulties when one endobag fails to fill properly due to constraints within calcified iliac vessels.

We wish Bi et al. well in their development of this new device and would be keen to look at it on completion.

\section{Compliance with Ethical Standards}

Conflict of Interest Francesco Torella has received professional fees, Research grants and educational fees from Endologix. Richard McWilliams is a paid consultant for Cook Medical and has received research funding from Endologix. Robert Fisher has received research and education grants from Endologix and education grants from Gore.

Ethical Approval This article does not contain any studies with human participants or animals performed by any of the authors.

\section{References}

1. Harrison GJ, Antoniou GA, Torella F, McWilliams RG, Fisher RK. Endovascular aneurysm repair and sealing (EVARS): a useful adjunct in treating challenging morphology. Cardiovasc Intervent Radiol. 2016;39(4):616-9.

2. Bi Y, Han X, Zhong H, Xu K, Ni Y. Re: "endovascular aneurysm sealing for the treatment of ruptured abdominal aortic aneurysms". J Endovasc Ther. 2015;22(6):956-7.

3. Bi Y, Han X, Xu K, Zhong H. Re: “endovascular aneurysm sealing for infrarenal abdominal aortic aneurysms: 30 day outcomes of 105 patients in a single centre". Eur J Vasc Endovasc Surg. 2015; 50(4):542.
Gareth J. Harrison

garethjamesharrison@yahoo.co.uk

1 Liverpool Vascular and Endovascular Service, The Royal Liverpool University Hospital, Prescot Street, Liverpool L7 8XP, UK 